\title{
SCIENTIFIC REPORTS

\section{OPEN Polypharmacy and oral antidiabetic treatment for type 2 diabetes characterised by drug class and patient characteristics: A Japanese database analysis}

\begin{abstract}
Takeshi Horii ${ }^{1}$, Makiko Iwasawa², Yusuke Kabeya ${ }^{3}$ \& Koichiro Atuda ${ }^{1}$
Polypharmacy (PP) occurs in patients with type 2 diabetes (T2DM) owing to multimorbidity. We evaluated concomitant PP and medication adherence in T2DM 3 years after initiation of administration of a hypoglycaemic agent using a nationwide claim-based database in Japan. Factors associated with medication PP and imperfect adherence were identified using multivariable logistic regression. PP was defined as using $\geq 6$ medications. Patients with proportion of days covered (PDC) of $<80 \%$ were defined as having poor medication adherence. A total of 884 patients were analysed. Multivariate analysis revealed that age, total number of consultations and body mass index (BMI) are factors that influence PP. Factors associated with PDC $<80 \%$ were 2-3, 4-5 and $\geq 6$ medications compared with 1 medication, male sex, $<17$ consultations and age $50-59$ and $\geq 60$ years compared with $<40$ years. In conclusion, older age, high total number of consultations and $B M I \geq 25 \mathrm{~kg} / \mathrm{m}^{2}$ are risk factors for PP. PP influenced good medication adherence at the end of the observation period.
\end{abstract}

In Japan, the number of patients with diabetes is steadily increasing; the National Health and Nutrition Examination Survey Japan 2018 estimated that 10 million people had diabetes ${ }^{1}$. Multiple medications are commonly prescribed for type 2 diabetes (T2DM). Management of hyperglycaemia, microvascular complications, macrovascular complications and medication side effects contribute to an increased number of medications among T2DM. Management of diabetes and its complications may improve target outcome but also contribute to the addition of unnecessary medications to the drug regimen ${ }^{2}$. Moreover, T2DM may seek medication therapy for diseases other than diabetes, such as hypertension or hyperlipidaemia, which can further contribute to the presence of polypharmacy (PP).

$\mathrm{PP}$ is associated with an increased risk for drug interactions, adverse events ${ }^{3}$, non-adherence and negative effects on activities of daily living and quality of life (QOL) ${ }^{3,4}$. Among hospitalised patients with T2DM in Japan, $64.6 \%$ report using 6 or more medications ${ }^{5}$. In the United States, 54\% of T2DM cases were found to be associated with $\mathrm{PP}^{6}$. Older adults with diabetes are at a greater risk of receiving $\mathrm{PP}$ than those without diabetes ${ }^{7}$. Adherence to hypoglycaemic medications is important for successful T2DM care and patient outcomes related to comorbidity management. However, it is difficult to manage diabetes treatment over a long term. A study conducted in the United States reported that medication adherence was low in asymptomatic patients with diabetes ${ }^{8}$ and that $32 \%$ of patients with T2DM failed to continue treatment ${ }^{9}$. In a previous study in Japan, poor medication adherence was shown to increase the risk of diabetic microangiopathy ${ }^{10}$.

Many factors affect adherence to T2DM medication, such as socio-economic factors, healthcare providers, depression, age and gender ${ }^{11}$. However, factors related to medication adherence in drug-naïve patients with

\footnotetext{
${ }^{1}$ Division of Clinical Pharmacy (Laboratory of Pharmacy Practice and Science I) and Research and Education Center for Clinical Pharmacy, Kitasato University School of Pharmacy, Sagamihara, Kanagawa, Japan. ${ }^{2}$ Division of Clinical Pharmacy (Laboratory of Drug Information) and Research and Education Center for Clinical Pharmacy, Kitasato University School of Pharmacy, Sagamihara, Kanagawa, Japan. ${ }^{3}$ Sowa Hospital, Department of Home Care Medicine, Sagamihara, Kanagawa, Japan. Correspondence and requests for materials should be addressed to T.H. (email: horiit@pharm.kitasato-u.ac.jp)
} 


\begin{tabular}{|c|c|}
\hline & Total cases $(n=884)$ \\
\hline Number of medications & $2.4 \pm 1.8$ \\
\hline Male (\%) & $797(90.2)$ \\
\hline Age (years) & $47.0 \pm 8.1$ \\
\hline Polypharmacy, n (\%) & $59(6.7)$ \\
\hline Smoking, n (\%) & $331(37.4)$ \\
\hline \multicolumn{2}{|c|}{ Drinking frequency, n (\%) } \\
\hline Every day & $180(20.4)$ \\
\hline Occasionally & $214(24.2)$ \\
\hline Scarcely & $321(36.3)$ \\
\hline Exercise habits, n (\%) & $128(14.5)$ \\
\hline HbAlc (\%) & $7.9 \pm 2.0$ \\
\hline $\mathrm{BMI}\left(\mathrm{kg} / \mathrm{m}^{2}\right)$ & $26.5 \pm 4.7$ \\
\hline \multicolumn{2}{|c|}{ Diabetes drug use rate, $\mathbf{n}(\%)$} \\
\hline DPP-4 inhibitor & $301(34.0)$ \\
\hline$\alpha \mathrm{GI}$ & $208(23.5)$ \\
\hline Glinide & $38(4.3)$ \\
\hline SU drug & $204(23.1)$ \\
\hline Biguanide & $235(26.6)$ \\
\hline Thiazolidine & $130(14.7)$ \\
\hline SGLT-2 inhibitor & $0(0)$ \\
\hline
\end{tabular}

Table 1. Patients' background characteristics. HbA1c, haemoglobin A1c; BMI, body mass index; DPP-4 inhibitor, dipeptidyl peptidase-4 inhibitor; $\alpha$ GI, $\alpha$-glucosidase inhibitor; SU, sulfonylurea; SGLT2 inhibitor, Sodium glucose cotransporter 2 inhibitor.
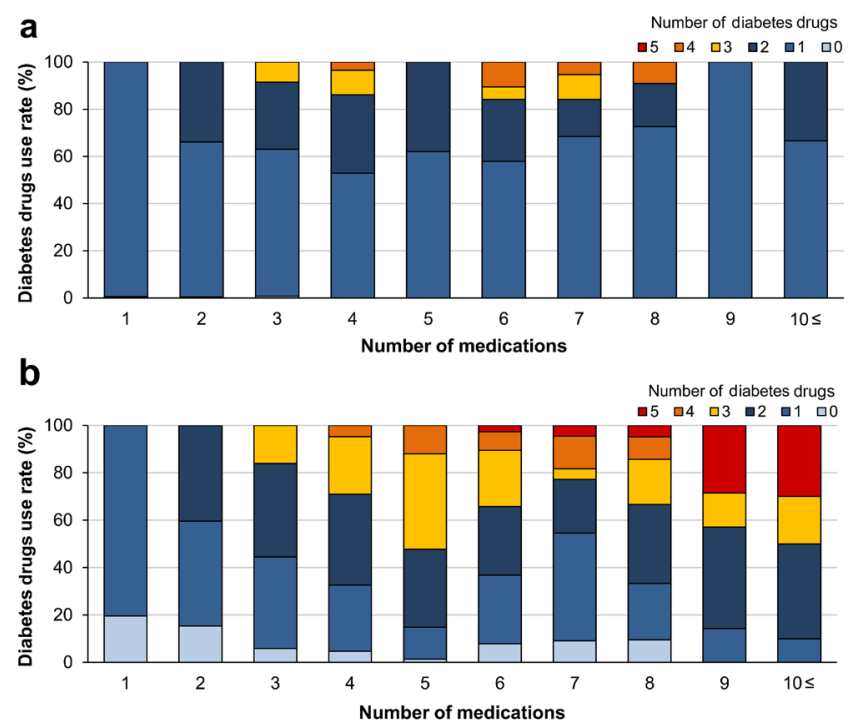

Figure 1. Number of medications and proportion of diabetes drugs. The number of medications and diabetes drugs at the start (a) and end (b) of the observation period are shown. At the start of the observation period, many diabetes drugs were used, whereas at the end of the observation period, the use of combination therapy showed an increasing trend.

T2DM have not been evaluated in Japan. Here we evaluated the use of oral hypoglycaemic medications and adherence to $\mathrm{PP}$ and medication, including risks for PP and imperfect adherence in drug-naïve patients with T2DM, using a large-scale receipt database.

\section{Results and Discussion}

The patients' background characteristics are presented in Table 1. A total of 884 patients were analysed. The mean patient age was $47.0 \pm 8.1$ years, and $90.2 \%$ patients were male. At the start of the observation period, the number of internal medications was $2.4 \pm 1.8$ and the rate of PP, defined $\geq 6$ internal medications, was $6.7 \%$. DPP- 4 inhibitors were the most commonly used hypoglycaemic medications, followed by biguanides, $\alpha$-glucosidase inhibitors and sulfonylureas. The number of medications and proportion of diabetes drugs are presented in Fig. 1. At the 


\begin{tabular}{|l|l|l|l|}
\hline \multicolumn{5}{|l|}{} & Odds ratio & $95 \%$ CI & P-value \\
\hline Sex: Male & 2.63 & $0.91-7.56$ & 0.073 \\
\hline Age (years) & \multicolumn{2}{l|}{} \\
\hline$<40(\mathrm{n}=144)$ & Reference & \\
\hline $40-49(\mathrm{n}=387)$ & 2.71 & $1.02-7.22$ & 0.046 \\
\hline $50-59(\mathrm{n}=301)$ & 2.96 & $1.10-7.99$ & 0.032 \\
\hline$\geq 60(\mathrm{n}=52)$ & 4.43 & $1.31-15.0$ & 0.017 \\
\hline Number of consultations in 3 years & \\
\hline $1-10(\mathrm{n}=207)$ & Reference & & \\
\hline $11-20(\mathrm{n}=174)$ & 5.56 & $1.56-20.0$ & 0.008 \\
\hline $21-30(\mathrm{n}=196)$ & 9.63 & $2.82-32.8$ & $<0.001$ \\
\hline$\geq 30(\mathrm{n}=307)$ & 11.6 & $3.52-38.5$ & $<0.001$ \\
\hline BMI $\left(\mathrm{kg} / \mathrm{m}^{2}\right) \geq 25$ & 1.71 & $1.04-2.80$ & 0.033 \\
\hline SU drug & 1.32 & $0.78-2.25$ & 0.309 \\
\hline
\end{tabular}

Table 2. Factors associated with polypharmacy at the end of the observation period, multiple logistic regression model. BMI, body mass index; SU, sulfonylurea.

\begin{tabular}{|l|l|l|l|}
\hline \multicolumn{5}{|l|}{} & Odds ratio & 95\% CI & P-value \\
\hline \multicolumn{4}{|l|}{ Number of medications at the end } \\
\hline $0.1(\mathrm{n}=372)$ & Reference & & \\
\hline $2.3(\mathrm{n}=261)$ & 0.34 & $0.22-0.51$ & $<0.001$ \\
\hline $4.5(\mathrm{n}=153)$ & 0.13 & $0.08-0.23$ & $<0.001$ \\
\hline$\geq 6(\mathrm{n}=98)$ & 0.18 & $0.10-0.34$ & $<0.001$ \\
\hline Sex: Male & 2.20 & $1.18-4.08$ & 0.013 \\
\hline Age $($ years) & \multicolumn{5}{|l}{} \\
\hline$<40(\mathrm{n}=144)$ & Reference & & \\
\hline $40-49(\mathrm{n}=387)$ & 0.74 & $0.44-1.23$ & 0.245 \\
\hline $50-59(\mathrm{n}=301)$ & 0.39 & $0.23-0.68$ & 0.001 \\
\hline$\geq 60(\mathrm{n}=52)$ & 0.37 & $0.15-0.89$ & 0.026 \\
\hline Total number of consultations/3 yrs.(times) & \multicolumn{2}{|l}{} \\
\hline$\geq 17(\mathrm{n}=597)$ & Reference & $15.6-47.0$ & $<0.001$ \\
\hline$<17(\mathrm{n}=287)$ & 27.1 & $0.49-1.10$ & 0.132 \\
\hline DPP-4 inhibitor & 0.73 & $0.40-0.96$ & 0.512 \\
\hline Biguanide & 0.62 & $0.63-1.86$ & 0.781 \\
\hline Thiazolidine & 1.08 & \\
\hline
\end{tabular}

Table 3. Factors influencing poor medication adherence, multiple logistic regression model. DPP-4 inhibitor, dipeptidyl peptidase-4 inhibitor.

end of the observation period, the frequency of the use of diabetes drugs and combination therapy tended to increase compared with those at the start of the observation period. Multivariate analysis with PP as the objective variable (Table 2) revealed statistically significant difference for age $40-49$ years (OR: $2.71,95 \%$ CI: $1.02-7.22$, $\mathrm{p}=0.046$ ), 50-59 years (OR: 2.96, 95\% CI: $1.10-7.99, \mathrm{p}=0.032$ ) and $\geq 60$ years (OR: 4.43, 95\% CI: $1.31-15.0$, $\mathrm{p}=0.017)$ compared with that for age $<40$ years; for total number of consultations during the 3-year observation period of 11-20 times (OR: 5.56, 95\% CI: 1.56-20.0, $\mathrm{p}=0.008$ ), 21-30 times (OR: 9.63, 95\% CI: 2.82-32.8, $\mathrm{p}<0.001$ ) and $\geq 30$ times (OR: $11.6,95 \%$ CI: 3.52-38.5, $\mathrm{p}<0.001$ ) compared with $1-10$ times and for body mass index (BMI) $\geq 25 \mathrm{~kg} / \mathrm{m}^{2}$ (OR: $1.71,95 \% \mathrm{CI}: 1.04-2.80, \mathrm{p}<0.033$ ).

The median PDC was $79.6 \%$ (31.7-96.1), and 50.2\% of the patients showed poor medication adherence. Receiver operating characteristic (ROC) analysis for the total number of consultations for PDC $<80 \%$ revealed that the cut-off value was 17 times during the 3 years (area under the curve: 0.86, sensitivity: 95.9\%, specificity: $60.6 \%$ ). Multivariate analysis with $\mathrm{PDC}<80 \%$ as the objective variable (Table 3 ) revealed that statistical significance was observed for $2-3$ medications (OR: $0.34,95 \%$ CI: $0.22-0.51, \mathrm{p}<0.001$ ), $4-5$ medications (OR: 0.13 , 95\% CI: 0.08-0.23, p < 0.001) and $\geq 6$ medications (OR: 0.18, 95\% CI: 0.10-0.34, p < 0.001) compared with 1 medication at the end of the observation; male sex (OR: 2.20, 95\% CI: $1.18-4.08, \mathrm{p}=0.013$ ); total number of consultations $<17$ times (OR: 27.1, 95\% CI: 15.6-47.0, $\mathrm{p}<0.001$ ) and age $50-<60$ years (OR: 0.39, 95\% CI: $0.23-0.68, \mathrm{p}<0.001$ ) and $\geq 60$ years (OR: $0.37,95 \%$ CI: $0.15-0.89, \mathrm{p}=0.026$ ) compared with $<40$ years.

The present study revealed that the risk factors for PP were older age, high total number of consultations and $\mathrm{BMI} \geq 25 \mathrm{~kg} / \mathrm{m}^{2}$. The rate of PP occurrence in the present study was $6.7 \%$, much lower than the approximately $50 \%$ rate previously reported ${ }^{12}$. The low rate may be attributed to our patients being younger ( $47.0 \pm 8.1$ years) 
and the presence of fewer comorbidities owing to the exclusion of patients with a history of prescription for hypoglycaemic medications. In the presence or absence of diabetes, the prevalence of hypertension and dyslipidaemia increases with age $\mathrm{e}^{11,13}$. In addition, the risk of T2DM onset increases during the middle years of life ${ }^{14}$; therefore, the duration of diabetes becomes longer as age increases. Simultaneously, the incidence of microangiopathy and its related disorders also increases ${ }^{15,16}$, thereby causing an increase in the risk of PP. Patients with a high total number of consultations are likely to have poor medical conditions and multiple complications. Thus, such patients may become polypharmic; however, further investigation is necessary to confirm this aspect. In overweight diabetic patients $\left(\mathrm{BMI} \geq 25 \mathrm{~kg} / \mathrm{m}^{2}\right)$, the accumulation of visceral fat is a known risk factor for cardiovascular events ${ }^{17}$; these events adversely affect blood pressure, lipid metabolism and blood glucose control. Risk of PP increases as drug therapies according to guidelines are initiated for each disease.

Next, we investigated the factors associated with poor medication adherence and found that OR was the lowest in the cohort with $4-5$ internal medications and that ORs with $\geq 6$ medications were only slightly increased. Several studies reported that T2DM with a high number of medications ${ }^{18}$ and complications ${ }^{19-22}$ had a positive association with adherence. These reports could partially explain why participants with 4-5 medications in the present study had a lower barrier for medication. On the other hand, medication adherence assumedly decreases as the number of internal medications increases and usage becomes more complicated; therefore, we believe that OR for good medication adherence can be achieved with $\geq 6$ medications, which is marginally higher compared with 4-5 medications.

In accordance with previous studies ${ }^{23-25}$, there was a distinct trend for increased adherence with increasing age in the present study. Male sex was a risk factor for poor medication adherence in the present study; nevertheless, the relationship between sex and medication adherence remains to be established. Males were reported to have a higher medication adherence than females ${ }^{23}$; however, several studies reported ${ }^{24-26}$ no sex-related differences in medication adherence. Analysis of the relationship between medication adherence and total number of consultations during the observation period resulted in a cut-off value of 17 times. Multivariate revealed found that $<17$ consultations during the observation period was a risk factor for poor medication adherence. Optimisation of prescriptions considering the patient's condition and the decreased frequency of guidance to medication adherence by pharmacists may have led to a lower frequency of consultations. Furthermore, it is possible that missed consultations or treatment interruptions are reflected in the lower frequency of consultation; therefore, this became a risk factor for poor medication adherence. Differences in medication adherence were expected depending on the class of the drugs because the methods of taking hypoglycaemic medications are diverse (e.g. once a day after meals or thrice a day immediately before every meal); however, there was no such differences were observed.

This study had several limitations. First, this was a retrospective study conducted using a large-scale receipt data; therefore, information and selection biases, may have affected the results. Second, owing to the small number of patients aged $\geq 65$ years, it was difficult to examine whether this age range was a risk factor for PP. Third, as most patients were male, further analyses are required to determine whether these findings apply to females as well. Finally, in this study, whether the medication was administered to inpatients or outpatients could not be distinguished. Medication management during hospitalisation may be performed by a medical practitioner, which may affect the outcomes of PDC.

Risk of PP is high in elderly patients and in patients with a high BMI. To avoid PP in such patients, evaluation and implementation of an appropriate drug therapy are necessary. Furthermore, higher numbers of consultations over a certain period are associated with a higher risk of PP. The good medication adherence observed in this study suggests that the prescriptions were optimised such that good medication adherence was possible even with PP. It is necessary to consider just how well patient prescriptions are optimised in clinical practice to determine if there is room for further improvement via intervention. The efficacy of telemedicine, such as online medical treatment to avoid increasing patient burden from frequent visits, should also be examined. Finally, although PP was speculated to be a risk factor for poor medication adherence, the contrary was observed in this study.

\section{Methods}

This retrospective, cohort study was conducted using large-scale receipt data, which houses a database of inpatient, outpatient and pharmacy claims from enrollees of employer-sponsored health plans throughout all prefectures in Japan ${ }^{27,28}$. Medical receipts contain information, such as disease code [Medical Information System Development Center standard disease master and International Classification of Diseases-10 (ICD-10)], prescription details and medical practices in addition to the patients' basic details. Patients were registered between May 2005 and January 2013. The inclusion criteria were as follows: (1) T2DM (ICD-10 code: E11) or diabetes (ICD-10 code: E14), (2) prescription history of hypoglycaemic medication and (3) capable of continuous follow-up observation for 3 years. The exclusion criteria were as follows: (1) type 1 diabetes, (2) age $<18$ years or $\geq 75$ years or (3) insulin and glucagon-like peptide-1 agonist use. Index date was defined as the start month of the prescribed diabetes medication.

Definition of polypharmacy. Of all medications, injections, external medicine, internal medicine with consistent usage and antibiotics to be used for $<7$ consecutive days were excluded. Although the number of medications has not been not strictly defined for PP, it was defined as $\geq 6$ internal medications for this study in accordance with previous reports in Japan. The number of internal medicines was defined as the number of drugs prescribed at the time of the completion of observations ${ }^{29}$.

Definition of medication adherence. Proportion of days covered (PDC) was calculated for each patient as an index of medication adherence. PDC was defined as the proportion of days for which the patient was prescribed and actually possessed hypoglycaemic medications during the observation period divided by the observation period. The formula used is as follows: 


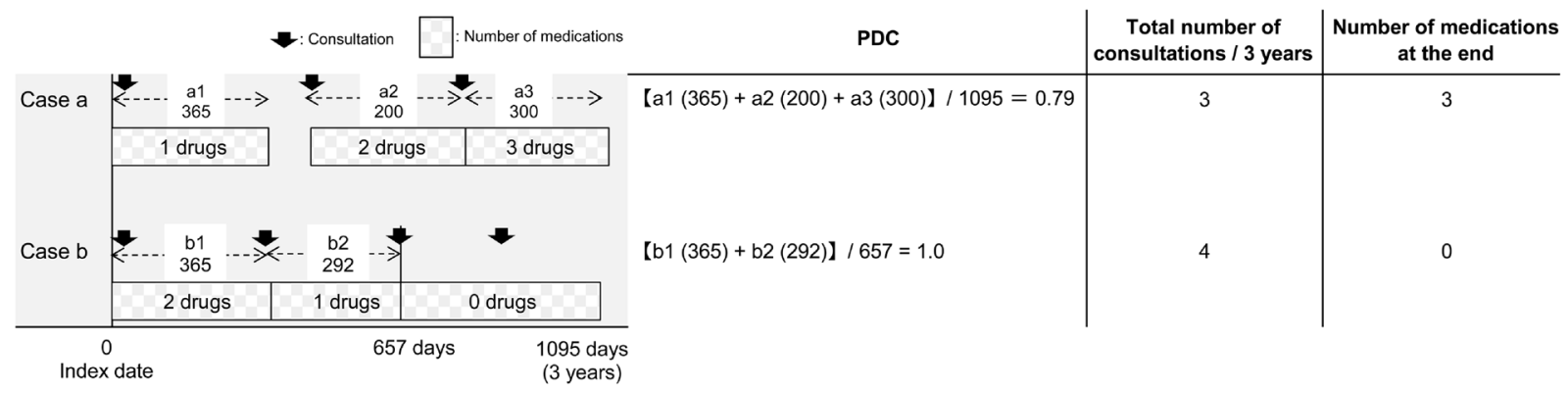

Figure 2. Proportion of days covered (PDC) based on the total number of consultations in 3 years and the number of medications at the end of the observation period. Case a: The prescription days of a1, a2 and a3 were added, and PDC was determined by dividing the number by 1095 days of the observation period. The number of drugs at the end of observation period corresponds to the number of drugs prescribed in a3. Case b: Since the medication use was completed at the third (b2) and subsequent consultations, the observation period for medication adherence was 657 days. The prescription days of b1 and b2 were added, and PDC was determined by dividing the number by 657 days of the observation period. The number of drugs at the end of observation period corresponds to the number of drugs prescribed in b3. Thus, the number of drugs at the end of the observation period is 0 .

$$
\begin{aligned}
\operatorname{PDC}(\%)= & {[\text { number of days possessing hypoglycaemic medications during the observation period }} \\
& \text { /observation period(days) }] \times 100
\end{aligned}
$$

PDC was calculated based on the total number of consultations in 3 years and the number of medications at the end of the observation period (Fig. 2). Patients with $\mathrm{PDC}<80 \%$ were defined as having poor medication adherence. Data on age and sex were collected using receipt information from the same year as that of the index date used to obtain the baseline patient backgrounds. Data on BMI were collected using receipt information from the same year as that of the observation start month. Haemoglobin A1c (HbAlc) data were collected from the receipt information of the same year as that of the observation start month and the receipt information 3 years after the observation start month. The total number of consultations for diabetes treatment during the 3-year observation period (total number of consultations) and the number of consultation days on which hypoglycaemic drugs were prescribed during the observation period were counted. Information on smoking and alcohol consumption frequencies was obtained from the results of the questionnaire administered to the patients at the time of medical examination in the same year as that of the start of observation.

Statistical analysis. Patient data were expressed as mean \pm S.D. With PDC $<80 \%$ as the objective variable, receiver-ROC analysis was conducted to calculate a cut-off value for the total number of consultations. Factors related to $\mathrm{PP}$ at the end of observation were examined using logistic regression analysis. Sex, age at baseline $(<40$, $40-49,50-59$ and $\geq 60$ years), HbA1c with a cut-off value of $7.0 \%$, BMI with a cut-off value of $25 \mathrm{~kg} / \mathrm{m}^{2}$, presence or absence of smoking, drinking frequency, presence or absence of exercise habits, use of hypoglycaemic agents for each medication and total number of consultations with the cut-off value calculated using ROC analysis were used as explanatory variables. Factors related to poor medication adherence were examined by adding the number of internal medications $(0-1,2-3,4-5$ or $\geq 6)$ to these explanatory variables at the end of the observation period. Based on the blood glucose control target values of diabetic patients in Japan, $7.0 \%$ was set as the cut-off value for $\mathrm{HbA} 1 \mathrm{c}$ and $25 \mathrm{~kg} / \mathrm{m}^{2}$, which is the target value for weight control, was set as the cut-off value for BMI. Univariate analysis was conducted for each explanatory variable. We performed univariate analysis on each of these predictor variables and multivariate analysis using factors for which $\mathrm{P}<0.2$ and calculated the odds ratio. Statistical analysis was performed using Stata 10 , and $\mathrm{P}<0.05$ was considered statistically significant.

Ethical considerations. Because we used unlinkable, anonymised data, this study did not require adherence to the Ethical Guidelines for Medical and Health Research Involving Human Subjects and the approval of the institutional review board committee of the Kitasato University and was, therefore, exempted from institutional review board consideration.

\section{Data Availability}

The datasets analysed during the current study are available from the corresponding author on reasonable request.

\section{References}

1. International Diabetes Federation. IDF Diabetes Atlas, http://www.diabetesatlas.org/across-the-globe.html (2015).

2. Boyd, C. M. et al. Clinical practice guidelines and quality of care for older patients with multiple comorbid diseases: Implications for pay for performance. JAMA. 294, 716-724 (2005).

3. Kojima, T. et al. Screening tool for older persons' appropriate prescriptions for Japanese: Report of the Japan Geriatrics Society Working Group on "Guidelines for medical treatment and its safety in the elderly". Geriatr. Gerontol. Int. 16, 983-1001 (2016). 
4. Elbert, S. H., Andrew, J. K., Kirstie, K. D., Warton, E. M. \& Ameena, T. A. The association between the number of prescription medications and incident falls in a multi-ethnic population of adult type-2 diabetes patients: the diabetes and ageing study. J. Gen. Intern. Med. 25, 141-146 (2010).

5. Horii, T., Kabeya, Y., Shimizu, J. \& Tomita, M. Study on risk of polypharmacy caused by diabetes. Jap. J. Pharmaceutic. Diabetes. 6, 179-187 (2017).

6. Grant, R., Nicole, W., Devita, R. P. H. \& Daniel, E. Polypharmacy and medication adherence in patients with type 2 diabetes. Diabetes Care. 26, 1408-1412 (2003).

7. Bauer, S. \& Nauck, M. A. Polypharmacy in people with Type 1 and Type 2 diabetes is justified by current guidelines-a comprehensive assessment of drug prescriptions in patients needing inpatient treatment for. diabetes-associated problems. Diabet. Med. 31, 1078-85 (2014).

8. Miller, N. H. Compliance with treatment regimens in chronic asymptomatic diseases. Am. J. Med. 102, 43-49 (1997).

9. Iglay, K. et al. Meta-analysis of studies examining medication adherence, persistence, and discontinuation of oral antihyperglycemic agents in type 2 diabetes. Curr. Med. Res. Opin. 31, 1283-1296 (2015).

10. Fukuda, H. \& Mizobe, M. Impact of nonadherence on complication risks and healthcare costs in patients newly diagnosed with diabetes. Diabetes Res. Clin. Pract. 123, 55-62 (2017).

11. Krass, I., Schieback, P. \& Dhippayom, T. Adherence to diabetes medication: a systematic review. Diabet. Med. 32, 725-737 (2015).

12. Jörgensen, T., Johansson, S., Kennerfalk, A., Wallander, M. A. \& Svärdsudd, K. Prescription drug use, diagnoses, and healthcare utilization among the elderly. Ann. Pharmacother. 35, 1004-1009 (2001).

13. Miura, K., Nagai, M. \& Ohkubo, T. Epidemiology of hypertension in Japan: where are we now? Circ. J. 77, 2226-2231 (2013).

14. Arai, H. et al. Serum lipid survey and its recent trend in the general Japanese population in 2000. J. Atheroscler. Thromb. 12, 98-106 (2005).

15. American Diabetes Association. Standards of medical care in diabetes-2019. Clin. Diab. 37, 11-34 (2019).

16. Kuusisto, J., Mykkänen, L., Pyörälä, K. \& Laakso, M. NIDDM and its metabolic control predict coronary heart disease in elderly subjects. Diabetes. 43, 960-967 (1994).

17. Bethel, M. A., Sloan, F. A., Belsky, D. \& Feinglos, M. N. Longitudinal incidence and prevalence of adverse outcomes of diabetes mellitus in elderly patients. Arch. Intern. Med. 167, 921-927 (2007).

18. Raum, E. et al. Medication non-adherence and poor glycaemic control in patients with type 2 diabetes mellitus. Diabetes Res. Clin. Pract. 97, 377-384 (2012)

19. Patel, A. et al. Intensive blood glucose control and vascular outcomes in patients with type 2 diabetes. N. Engl. J. Med. 358, 2560-2572 (2008).

20. Jamous, R. M. et al. Adherence and satisfaction with oral hypoglycemic medications: a pilot study in Palestine. Int. J. Clin. Pham. 33, 942-948 (2011).

21. Kreyenbuhl, J. et al. Does adherence to medications for type 2 diabetes differ between individuals with vs without schizophrenia? Schizophr. Bull. 36, 428-435 (2010).

22. Sufiza, A. N., Ramli, A., Islahudin, F. \& Paraidathathu, T. Medication adherence in patients with type 2 diabetes mellitus treated at primary health clinics in Malaysia. Patient Prefer. Adherence. 7, 525-530 (2013).

23. Curkendall, S. M., Thomas, N., Bell, K. F., Juneau, P. L. \& Weiss, A. J. Predictors of medication adherence in patients with type 2 diabetes mellitus. Curr. Med. Res. Opin. 29, 1275-1286 (2013).

24. Hertz, R. P., Unger, A. N. \& Lustik, M. B. Adherence with pharmacotherapy for type 2 diabetes: a retrospective cohort study of adults with employer-sponsored health insurance. Clin. Ther. 27, 1064-1073 (2005).

25. Donnan, P. T., MacDonald, T. M. \& Morris, A. D. Adherence to prescribed oral hypoglycaemic medication in a population of patients with Type 2. diabetes: a retrospective cohort study. Diabet. Med. 19, 279-284 (2002).

26. Michel, T. et al. Medication adherence in type 2 diabetes: the ENTRED study 2007, a French population-based study. PLoS One. 7 , e32412 (2012).

27. Kimura, S., Sato, T., Ikeda, S., Noda, M. \& Nakayama, T. Development of a database of health insurance claims: standardization of disease classifications and anonymous record linkage. J. Epidemiol. 20, 413-419 (2010).

28. Nakaoka, S. et al. Echocardiography for the detection of valvulopathy associated with the use of ergot-derived dopamine agonists in patients with Parkinson's disease. Intern. Med. 50, 687-694 (2011).

29. Kojima, T. et al. High risk of adverse drug reactions in elderly patients taking six or more drugs: analysis of inpatient database. Geriatr. Gerontol. Int. 112, 761-762 (2012).

\section{Acknowledgements}

This work was supported by the Japan Diabetes Society Medical Staff Training and Research Fund.

\section{Author Contributions}

Takeshi Horii designed the study, and wrote the initial draft of the manuscript. Yusuke Kabeya contributed to analysis and interpretation of data, and assisted in the preparation of the manuscript. Koichiro Atuda and Makiko Iwasawa have contributed to data collection and interpretation, and critically reviewed the manuscript. All authors approved the final version of the manuscript, and agree to be accountable for all aspects of the work in ensuring that questions related to the accuracy or integrity of any part of the work are appropriately investigated and resolved.

\section{Additional Information}

Competing Interests: The authors declare no competing interests.

Publisher's note: Springer Nature remains neutral with regard to jurisdictional claims in published maps and institutional affiliations.

Open Access This article is licensed under a Creative Commons Attribution 4.0 International License, which permits use, sharing, adaptation, distribution and reproduction in any medium or format, as long as you give appropriate credit to the original author(s) and the source, provide a link to the Creative Commons license, and indicate if changes were made. The images or other third party material in this article are included in the article's Creative Commons license, unless indicated otherwise in a credit line to the material. If material is not included in the article's Creative Commons license and your intended use is not permitted by statutory regulation or exceeds the permitted use, you will need to obtain permission directly from the copyright holder. To view a copy of this license, visit http://creativecommons.org/licenses/by/4.0/.

(C) The Author(s) 2019 